\title{
Dietary therapy: a new strategy for management of chronic pelvic pain
}

\author{
Francesco Sesti $^{1 *}$, Talia Capozzolo ${ }^{1}$, Adalgisa Pietropolli ${ }^{1}$, Marco Collalti ${ }^{1}$, Maria Rosa Bollea $^{2}$ \\ and Emilio Piccione ${ }^{1}$ \\ ${ }^{1}$ Endometriosis Center, Section of Gynecology and Obstetrics, Department of Surgical Sciences, Tor Vergata University \\ Hospital, Rome, Italy \\ ${ }^{2}$ Section of Clinical Nutrition, Department of Internal Medicine, Tor Vergata, University Hospital, Rome, Italy
}

\section{Abstract}

Chronic pelvic pain (CPP) can be identified as a chronic nociceptive, inflammatory and neuropathic pain characterised by spontaneous pain and an exaggerated response to painful and/or innocuous stimuli. This pain condition is extremely debilitating and usually difficult to treat. Currently, the main approaches to treatment include counselling supported by reassuring ultrasound scanning or psychotherapy, attempting to provide reassurance using laparoscopy to exclude serious pelvic pathology, hormonal therapy and neuroablative treatment to interrupt nerve pathways. Dietary supplementation has been suggested as a means to treat chronic medical illnesses that are poorly responsive to prescription drugs or in which therapeutic options are limited, costly or carry a high side-effect profile. A comprehensive search of the PubMed database was performed using the search terms 'chronic pelvic pain', 'oxidative stress', 'antioxidants' and 'dietary therapy'. The systematic review focuses on both randomised and non-randomised controlled trials from 2005 onwards, in which CPP was the end point. Given the complexity and not well-understood aetiology of CPP, its treatment is often unsatisfactory and limited to partial symptom relief. Dietary therapy with antioxidants improves function of the immune system and in fighting free radical damage. Agents with antioxidant activity are able to improve CPP without undesired effects and any important metabolic changes associated with hormonal suppression therapy. In conclusion, dietary therapy with antioxidants could be considered as a new effective strategy in the long term for CPP, and may be better accepted by patients. Further randomised trials with larger series and long-term follow-up to confirm these observations are needed.

\section{Key words: Pelvic pain: Oxidative stress: Antioxidants: Dietary therapy}

\section{Introduction}

The present review comprehensively explores the literature for evidence of chronic pelvic pain (CPP) in women, such as nociceptive, inflammatory and neuropathic pain. Based on these findings, the focus of the present review was to provide some insight into the potential role and clinical use of dietary supplementation in the management of CPP. The present systematic review focuses on both randomised and non-randomised controlled trials from 2005 onwards, in which CPP was the end point. To identify relevant studies we performed a comprehensive search of the PubMed database, using the search terms 'chronic pelvic pain', 'oxidative stress', 'antioxidants' and 'dietary therapy'.

\section{Epidemiology and impact on quality of life}

Women with CPP present a diagnostic and therapeutic challenge. Pelvic pain is a major health problem and affects between 4 and 39\% of women and accounts for 10-40\% of all out-patient gynaecological visits ${ }^{(1)}$. A quarter of women with CPP miss nearly $15 \mathrm{~h}$ of work per month, which accounts for a $\$ 14$ billion loss in productivity per year ${ }^{(2)}$. The estimated combined cost of CPP owing to loss of productivity, diagnosis, and medical and surgical treatment is $\$ 39$ billion annually ${ }^{(2)}$.

CPP is a non-menstrual pelvic pain of 6 or more months' duration that is severe enough to cause functional disability or require medical or surgical treatment ${ }^{(3)}$. Pelvic pain is a syndromic complex; universally it is the unpleasantness of the experience that characterises pain, and this makes pain an emotional experience, not simply a sensory experience ${ }^{(4)}$. So, the constant presence of pain may be responsible for affective changes in dynamics: family, social and sexual.

In a study by Sepulcri \& do Amaral ${ }^{(5)}$, the prevalence of anxiety was 73 and $37 \%$ in pelvic pain and control subjects, respectively, as evaluated by the Spielberger State-Trait Anxiety Inventory and the Hamilton Rating Scale for Anxiety. The prevalence of depression was 40

Abbreviations: COX, cyclo-oxygenase; CPP, chronic pelvic pain; NGF, nerve growth factor.

*Corresponding author: Dr Francesco Sesti, fax +3906 20902 921, email Francesco.Sesti@uniroma2.it 
and $30 \%$ in the pelvic pain and control groups, respectively, as evaluated by the Beck Depression Inventory and the Hamilton Rating Scale for Depression. Patients with higher anxiety and depression scores also presented lower quality-of-life scores ${ }^{(5)}$.

\section{Aetiology}

Specific causes of CPP may include many disorders of the reproductive tract such as pelvic endometriosis, interstitial cystitis, adhesions ( $40 \%$ of cases), pelvic congestion syndrome (20\% of cases), pelvic inflammatory disease, or diseases of the gastrointestinal system, urological organs, musculoskeletal system and psychoneurological system $^{(6,7)}$. Moreover, it has been postulated that an association may exist between CPP and sexual/physical abuse ${ }^{(8)}$. However, as confirmed by Sharma et al. in a recent study, CPP is usually associated with pelvic pathology and requires laparoscopy before it can be attributed to a psychosomatic cause $^{(7)}$.

Up to half of CPP cases have been found to be associated with either genitourinary symptoms or symptoms of irritable bowel syndrome ${ }^{(9)}$. Women with CPP often develop visceral and somatic hyperalgesia as a result of visceral hypersensitivity arising from the gastrointestinal and urinary tracts and the reproductive organs ${ }^{(10)}$. Furthermore, interstitial cystitis and irritable bowel syndrome may be associated with endometriosis, dysmenorrhoea, vulvodynia and adhesions through the recruitment of additional neural pathways, thereby substantially complicating the $\operatorname{diagnosis}^{(11)}$.

\section{Diagnosis}

A thorough history, clinical examination and imaging studies provide considerable information but are insufficient to arrive at a diagnosis in all cases. There are many reports in the literature that $\mathrm{CPP}$ is best investigated laparoscopically before any treatment is planned ${ }^{(7,12)}$. It is important to remember that a negative laparoscopy is not synonymous with no diagnosis or no disease. More discriminative use of laparoscopy, carefully based on the patient's history, physical examination, laboratory, and imaging findings, might decrease the rate of negative laparoscopies from 39 to $4 \%{ }^{(13)}$.

\section{Pathophysiology}

Fig. 1 shows the proposed mechanism of action for endometriosis-related CPP.

\section{Nociceptive pelvic pain}

Nociceptive pain occurs in response to a noxious stimulus that initiates the nociceptive pathway. The clinical characteristics of visceral pelvic pain include that it is not always

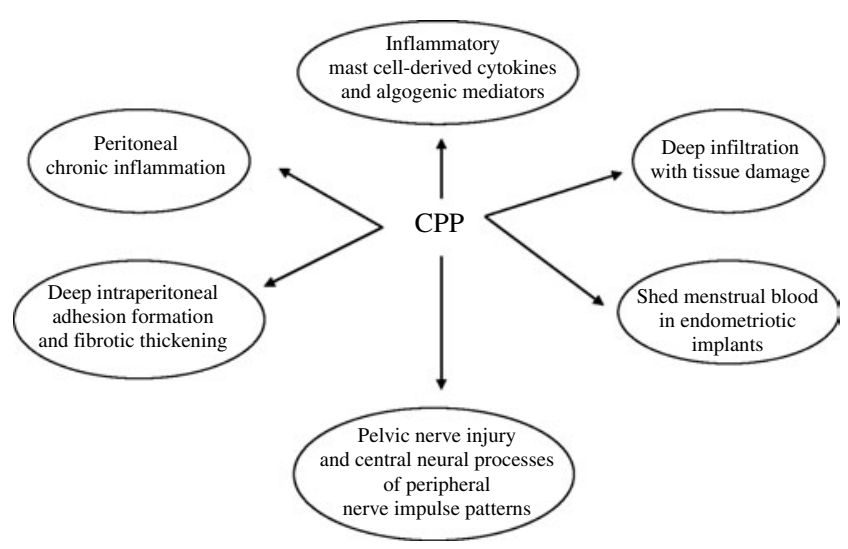

Fig. 1. Proposed mechanism of action for endometriosis-related chronic pelvic pain (CPP).

linked to injury and thus may be functional; it frequently results in somatic referral of pain, possibly because of central convergence of visceral and somatic afferents; it tends to be diffuse or poorly localised, probably because of the low concentration of nociceptive afferents within viscera $^{(14)}$.

Many nociceptors in viscera are silent nociceptors that do not normally respond to intense mechanical or thermal stimuli, but when there is inflammation of the surrounding tissue they become sensitised and respond to several stimuli $^{(15)}$. Moreover, the severity of visceral pain does not always reflect the severity of the pain-generating condition, in acute and also chronic visceral pelvic pain ${ }^{(15)}$.

The nerves that function as nociceptors are primarily $\mathrm{C}$ and A fibres ${ }^{(13)}$.

There is clear evidence that mechanisms of nociceptive pain are implied in CPP and that peripheral and central sensitisation leads to abnormal perception of both painful and non-painful stimuli contributing to visceral hyperalgesia and allodynia ${ }^{(13)}$. Temporal and spatial summation of pain stimuli is also thought to be important in the development of hyperalgesia at the site of an irritated organ that develops as a result of enhanced excitability of respective neuronal soma within the dorsal root ganglia ${ }^{(16)}$.

The major evidence for nociceptive pelvic pain as a trigger mechanism for CPP is represented by endometriosis and comes from clinical trials of laparoscopic surgical ablation or excision of endometriotic lesions ${ }^{(17)}$. In this context it is important to recognise that there was incomplete relief of pain after surgical treatment of endometriosis and that many women continued to have some degree of pelvic pain ${ }^{(13)}$.

\section{Inflammatory pelvic pain}

Inflammatory pain is due to the response to tissue injury and the resulting inflammatory process; it may be a normal pain, but in some cases it becomes chronic or persistent and represents a pathological pain mechanism ${ }^{(13)}$. The most frequent pelvic cause of the CPP-related inflammatory process is endometriosis ${ }^{(18)}$. 
Some research has confirmed that endometriotic implants develop a sensory and sympathetic nerve supply both in rats and in humans ${ }^{(19,20)}$. The nerve invasion by means of perineural and endoneural invasion, as well as the presence of degranulating mast cells near nerve structures, could be responsible for the inflammatory pain and hyperalgesia ${ }^{(21)}$. After tissue damage, a variety of immune mediators are released, which exert algesic actions by acting directly on nociceptors, or indirectly via the release of other mediators, most notably prostanoids ${ }^{(22)}$.

There is increasing knowledge of the intracellular cascades that are activated in nociceptors by mediators such as TNF- $\alpha$, IL-1 $\beta$, IL-6, NO, nerve growth factor (NGF) and cyclo-oxygenase (COX)-2, which ultimately either activate or sensitise these neurons ${ }^{(22)}$.

Mast cells, residing in the nerve, are the first cells to be activated and contribute to the recruitment of neutrophils and macrophages. In the setting of inflammation, mast cells degranulate and release their inflammatory mediators and may activate or sensitise primary nociceptive neurons ${ }^{(22)}$. These initial events promote the recruitment of T-cells, which reinforce and maintain inflammatory reactions.

Deregulation of neural transmission appears be produced by neurotransmitters, neuromodulators, neuropeptide hormones or paracrine regulators including the neuroendocrine system cells stained with specific immunohistochemical markers such as neuron-specific enolase, synaptophysin, chromogranin A and cytokeratin ${ }^{(23)}$.

In modulation of acute inflammation, monocyte chemotactic protein $1 /$ chemokine (C-C motif) ligand 2 (MCP-1/ CCL2) appears to be at least one of the factors responsible for this increase of activated macrophages, stimulating macrophages to secrete growth factors and cytokines, increasing levels of many inflammatory cytokines ${ }^{(24)}$.

After peripheral nerve injury, the site of damage is typified by the activation of resident immune cells, and recruitment and proliferation of non-neuronal elements, which release factors (for example, TNF- $\alpha$, IL-1, IL-6, C-chemokine ligand 2, histamine, $\mathrm{PGE}_{2}$ and $\mathrm{NGF}$ ) that initiate and maintain sensory abnormalities after injury. These factors may either induce activity in axons or are transported retrogradely to cell bodies in the dorsal root ganglia, where they may alter gene expression of the neurons ${ }^{(22)}$.

TNF- $\alpha$ is a cytokine that has an essential role in inflammatory process and in CPP. As well documented, TNF- $\alpha$ stimulates the expression of $\mathrm{PG}$ synthase-2, which in turn increases the production of $\mathrm{PGE}_{2}$ and $\mathrm{PGF}_{2 \alpha}$, an indirect mechanism by which TNF- $\alpha$ may cause inflammatory pain ${ }^{(25)}$.

Between them, IL-1, IL-6 and IL-8 have many biological functions such as inducing the synthesis of inflammatory prostaglandins and promoting fibroblast proliferation, collagen deposition and fibrinogen formation, which can contribute to the pathophysiology of chronic painful symptoms ${ }^{(26)}$.

Recent evidence has revealed that NGF, a cytokine produced by Schwann cells, keratinocytes, fibroblasts, $\mathrm{T}$ lymphocytes and macrophages, regulated by oestrogen and progesterone, involved in the extension and maintenance of sympathetic and primary sensory nerves, is greater expressed in pelvic symptomatic endometriotic lesions ${ }^{(27)}$ NGF promotes sprouting of nociceptors, increases the number of sensory neurons, and is known to contribute to persistent inflammatory and also neuropathic pain. NGF induces the expression of substance $\mathrm{P}$ and calcitonin gene-related peptide, which are neuropeptides involved in the modulation of central pain transmission ${ }^{(27)}$.

In the peritoneal fluid of women with CPP elevated levels of prostaglandins have been found, especially $\mathrm{PGE}_{2}$ and $\mathrm{PGF}_{2 \alpha}$. These prostaglandins are mediators of inflammation, as well as direct generators of pain, activating nerve endings to pain, and trigger the release of other algesic mediators, such as serotonin and histamine from other cells and afferent nerves ${ }^{(28)}$.

The increase of $\mathrm{PGE}_{2}$ is due to the up-regulation of COX-2. This is the rate-limiting enzyme that catalyses the initial step in the formation of prostaglandins from arachidonic acid. COX-2 expression is induced by a number of inflammatory, mitogenic and physical stimuli and is stimulated by oestradiol ${ }^{(29)}$. So, a positive feedback loop appears to be present in women with endometriosisrelated painful symptoms that supports the continuous formation of oestradiol and $\mathrm{PGE}_{2}$.

\section{Neuropathic pelvic pain}

Neuropathic pain is usually accompanied by nerve injury or prolonged neuronal pressure but, additionally, the activation of cells involved in the immune response may contribute indirectly to the development of CPP and frequently leads to a chronic pain state by neural plasticity and central sensitisation ${ }^{(30)}$. Also, visceral cross-sensitisation is believed to be due to increased, persistent nociceptive input from inflamed reproductive system organs that sensitise neurons, that receive input not only from the inflamed reproductive organs, but also from unaffected visceral organs by a phenomenon called convergent input ${ }^{(31)}$.

Moreover, the nociceptive memory manifests itself most prominently as post-injury sensitisation; that is, after tissue damage, pain that results from subsequent stimulation is exaggerated and prolonged and can be initiated by lowintensity stimuli.

Atwal et al. studied nerve fibre concentration, microneuroma formation and perivascular nerve proliferation within the lower segment of the uterus and found that changes observed in innervation of the endometrium were much more prominent in uteri from women with endometriosis and with CPP than from women without either ${ }^{(32)}$.

\section{Chronic pelvic pain and oxidative stress}

Reactive oxygen species are a double-edged sword; they serve as key signal molecules in physiological processes, 
but also they have a role in pathological processes involving the reproductive tract $^{(33)}$.

Given the limited data and complex environment of the peritoneal cavity, it is unclear when and why oxidative stress may occur in relation to CPP. In addition, circulating levels of oxidative stress due to other causes such as persistent organic compounds and exposure to environmental toxicants, for example, 2,3,7,8-tetrachlorodibenzo- $p$-dioxin and heavy metals, may further induce symptomatic pelvic diseases.

It has been suggested that in women with endometriosis-related CPP there is an increased peritoneal level of lipid peroxidation markers and their by-products in a macrophage-rich inflammatory milieu. This finding is associated with an altered expression of pro-oxidants and a drop in antioxidant levels, with alterations in free radical detoxification pathways, consequentially ${ }^{(34)}$.

As a potential inducer of $\mathrm{NF}-\kappa \mathrm{B}$, which activates genes involved in cell adhesion, secretion of inflammatory cytokines, and recruitment of macrophages, oxidative stress may help to trigger the chain of events that leads to the development of symptomatic pelvic diseases ${ }^{(35)}$.

Oxidative stress induces a cytotoxic effect by peroxidation of membrane phospholipids, increased cell membrane permeability, loss of membrane integrity, enzyme activation, structural damage to DNA and cell death ${ }^{(36)}$. The presence of activated macrophages and LDL together may lead to oxidation. Increased number and activity of polymorphonuclear leucocytes and oxidative stressinduced macrophages lead to an increased production of reactive oxygen species with higher levels of lipid peroxide accompanied by the release of more cytokines and other immune mediators, such as $\mathrm{NO}^{(37)}$.

Various cytokines released by abnormal immune responses might stimulate macrophages and/or endometrial cells to persistently produce a large amount of $\mathrm{NO}^{(38)}$

Peritoneal macrophages express higher levels of the inducible NO synthase isoform, persistently marked throughout the menstrual cycle, in contrast to cyclic variations in healthy subjects, and produce more $\mathrm{NO}$ in response to immune stimulation in vitro ${ }^{(39)}$. $\mathrm{NO}$ is a free radical initially implicated in low-grade inflammation, while elevated peritoneal NO levels are consistent with the increased number and activity of macrophages ${ }^{(40)}$.

Elevated levels of the marker of lipid peroxidation lysophophatidyl choline, a potent chemotactic factor for monocytes/T-lymphocytes, were seen in the peritoneal fluid of women with endometriosis ${ }^{(28)}$. Non-terminal oxidation may have a role in the pathophysiology of endometriosis. Minimally oxidised LDL is present in the peritoneal fluid of women with endometriosis in place of the terminally oxidised $\mathrm{LDL}^{(41,42)}$. The ratio of lysophosphatidyl choline, a breakdown product of oxidised-LDL, to phosphatidyl choline suggests minimally oxidised LDL rather than oxidised LDL.
Retrograde menstruation is likely to carry highly pro-oxidant factors into the peritoneal cavity. In addition, it is likely that damaged blood cells releasing metal ions as well as haeme and Fe, cell debris transplanted into the peritoneal cavity by menstrual reflux, and apoptotic endometrial cells would provide an ideal target for the recruitment of peritoneal fluid monocytes ${ }^{(43)}$.

On the other hand, expression of antioxidant enzymes (superoxide dismutase, glutathione peroxidase, catalase) and non-enzymic antioxidants (taurine, vitamin C, vitamin E) appears to be reduced in the peritoneal fluid of patients with developing CPP compared with fertile symptom-free control women ${ }^{(28)}$. These enzymes play an important role in converting reactive oxygen species to water to prevent overproduction of reactive oxygen species, and in the breakdown of free radicals. Thereby, antioxidants might prevent the pathological effects of oxidative stress that are exerted by various mechanisms.

\section{Therapeutic approach}

\section{Conventional treatments}

Given the complexity and not well-understood aetiology of CPP, its treatment is often unsatisfactory and limited to partial symptom relief.

Currently, the main approaches to treatment include counselling supported by reassuring ultrasound scanning or psychotherapy, attempting to provide reassurance using laparoscopy to exclude serious pelvic pathology, hormonal therapy, and neuroablative treatment to interrupt nerve pathways ${ }^{(44)}$.

The treatment of CPP can focus on treating with medical or surgical interventions the underlying disease that might be a cause or a contributor to CPP, or on treating the pain itself, or on both ${ }^{(45)}$.

Different pharmacological profiles and mechanisms to interrupt or decrease the transmission of pain information might be beneficial to decrease pain.

Clinical experience suggests that non-narcotic analgesics, including acetaminophen, acetylsalicylic acid and non-steroidal anti-inflammatory drugs are the first-line therapy for pain relief treating neural and biochemical pathways of $\mathrm{CPP}^{(44)}$. The potential of side effects with analgesic drugs, especially with chronic use, are significant, and careful observation is important.

Only after all other reasonable attempts at pain control have failed and when persistent pain is the major impediment to improved function, tricyclic antidepressants, serotonin-noradrenaline reuptake inhibitors, and, as a last resort, opioids for CPP should be considered ${ }^{(45)}$. Opioids are the major category of analgesics with central activity used for chronic pain, whereas their use is controversial.

However, in a Cochrane analysis of treatments for CPP, only progestogen therapy such as medroxyprogesterone acetate (50 mg once daily) and lysis of severe deep 
adhesions were found to be associated with an improved outcome ${ }^{(46)}$.

Finally, a recent pilot study proposed the combination of palmitoylethanolamide and polydatin as a support for medical therapies to treat CPP associated with endometriosis to obtain pain relief and a reduction in the analgesic drugs usually employed for the control of pain ${ }^{(47)}$. It has been reported that palmitoylethanolamide and its derivates play a role in controlling inflammation associated with mast cell activation, whereas polydatin is a resveratrol with wellknown antioxidant and anti-chemotactic activities, as well as a regulator of aspecific leucocyte activation ${ }^{(48,49)}$.

\section{Dietary therapy}

On account that potential benefit of medical therapy must be balanced against potential risk, dietary supplementation has been suggested to treat chronic medical illnesses that are poorly responsive to prescription drugs or in which therapeutic options are limited, costly or carry a high side effect profile ${ }^{(50)}$.

Over the past decade, many studies have provided evidence that higher intakes of fruit and vegetables, rich in antioxidants, among other micronutrients, improve the function of the immune system and fight free radical damage $^{(51)}$.

In the USA, dietary supplements are currently governed by the Food and Drug Administration under the Dietary Supplement Health and Education Act. The act defines a supplement as 'a product (other than tobacco) intended to supplement the diet that bears or contains one or more of the following: a vitamin, mineral, herb or other botanical, amino acid, a dietary substance used by man to supplement the diet by increasing the total dietary intake or a concentrate, metabolite, constituent, extract or combination of any of the ingredients described above ${ }^{(52)}$. Unfortunately, there is a deficit of well-designed, randomised, controlled trials to evaluate the efficacy and safety of complementary dietary therapy to manage CPP.

There is clear evidence for the effectiveness of $n$ - 3 fatty acids, vitamin $\mathrm{E}$, vitamin $\mathrm{B}_{1}$, vitamin $\mathrm{B}_{3}$ and $\mathrm{Mg}$ in pain relief and the need for less additional medication in cyclic pelvic pain by a decreased release of prostaglandins, primarily $\mathrm{PGE}_{2}$ and $\mathrm{PGF}_{2 \alpha}$, which cause inflammation, pain and spastic uterine contractions ${ }^{(53,54)}$.

Inhibitors of COX enzyme function, such as vitamins and $n$-3 fatty acids, can block prostaglandins and thromboxane $\mathrm{A}_{2}$ biosynthesis released from plasma membrane phospholipids by phospholipase $\mathrm{A}_{2}$ and subsequent downstream events $^{(55)}$. Moreover, oestrogen and progesterone withdrawal leads to the stimulation of $\mathrm{PGF}_{2 \alpha}$ production via reactive oxygen species-induced $\mathrm{NF}-\kappa \mathrm{B}$ activation ${ }^{(35)}$.

So, manipulation of dietary PUFA composition demonstrably affects the pro-inflammatory activities of many cell types involved in the immune response and inflammatory reactions ${ }^{(56)}$.
Table 1. Food n-3:n-6 fatty acid ratios

\begin{tabular}{|c|c|c|}
\hline Food & $n-3(g)$ & $n-6(g)$ \\
\hline \multicolumn{3}{|l|}{ Fatty fish (per $100 \mathrm{~g})$} \\
\hline $\begin{array}{l}\text { Salmon, fresh or tinned mackerel, } \\
\text { fresh or smoked herring }\end{array}$ & $2 \cdot 2$ & - \\
\hline \multicolumn{3}{|l|}{ Pressed vegetable oils (per $10 \mathrm{~g}$ ) } \\
\hline Maize oil & - & 5 \\
\hline Sesame oil & - & $4 \cdot 5$ \\
\hline Soya oil & 0.8 & 5 \\
\hline Sunflower-seed oil & - & 6 \\
\hline Linseed oil or $40 \mathrm{~g}$ linseeds & $5 \cdot 8$ & $1 \cdot 4$ \\
\hline Wheat germ oil & 0.5 & 5 \\
\hline Olive oil & - & 1 \\
\hline \multicolumn{3}{|l|}{ Dried fruit } \\
\hline $\begin{array}{l}\text { Shelled walnuts (per } 20 \mathrm{~g} ; \\
\text { five medium-sized walnuts) }\end{array}$ & $1 \cdot 32$ & 5 \\
\hline $\begin{array}{l}\text { Groundnuts, pistachios or } \\
\text { almonds (per } 10 \mathrm{~g} \text { ) }\end{array}$ & - & 1 \\
\hline \multicolumn{3}{|l|}{ Legumes } \\
\hline Tinned chick peas (per $100 \mathrm{~g}$ ) & - & 2 \\
\hline
\end{tabular}

PUFA supplementation has important effects on the synthesis and biological activity of prostaglandins and cytokines such as IL-1, IL-2, IL-6, TNF and interferon, and might be useful in the management of chronic painful symptoms by reducing the inflammatory response and modulating cytokine network ${ }^{(57)}$.

In the dynamic structures of the cell membrane, the intrinsic structural synergy via ester bonds of fatty acids such as DHA and EPA, phospholipids and antioxidants in the 'triple cell membrane synergy' steadies and increases membrane fluidity, improving its biochemical performance $^{(58)}$.

Healthy, non-inflammatory eicosanoid balance is maintained throughout the body by way of a homeostatic balance between $n-3$ and $n-6$ fatty acids in cell membranes. Table 1 shows $n-6: n-3$ fatty acid ratios in food.

In the presence of high $n-6: n-3$ PUFA ratios of dietary intake, biosynthesis of their metabolites steadies a prominent production of 2-series prostaglandins ( $\mathrm{PGE}_{2}, \mathrm{PGF}_{2 \alpha}$ ), thromboxane $\mathrm{A}_{2}$ and 4-series leucotrienes, which also are involved in the pathogenesis of pelvic pain, in contrast to high n-3:n-6 PUFA ratios ${ }^{(56)}$.

In addition, $\mathrm{PGE}_{2}$ is thought to be a potent inducer of aromatase activity in endometriotic stromal cells. Of note, peritoneal macrophages from women with CPP release significantly more prostaglandins.

Likewise, $\mathrm{Mg}$ inhibits the biosynthesis of $\mathrm{PGF}_{2 \alpha}$, as well as having a role in myometrial relaxation and vasodilatation, and vitamin $\mathrm{B}_{6}$ is involved in the production of $\mathrm{PGE}_{2}$.

Moreover, a diet based on vitamin $\mathrm{B}$, vegetables, fibres and antioxidants decreases oestrogenic state-related body fat excess implicated in the oestrogen-dependent growth of endometriotic tissue ${ }^{(56)}$.

In actual fact, dietary supplementation induces enzymes of oestradiol metabolism such as the up-regulation of $17-\beta$-hydroxysteroid dehydrogenase (types 1 and 7) and sulfatase; the subsequent defective formation and 
metabolism of steroid hormones are responsible for the promotion and development of endometriosis ${ }^{(59)}$.

It may be supposed that nutritional intake has significant influence whenever there is an imbalance between the production of reactive oxygen species and aberrant expression of antioxidant systems, improving painful symptoms, without undesired effects and any important metabolic changes associated with hormonal therapy ${ }^{(54-59)}$.

In this context, $\alpha$-tocopherol is considered to be the most important vitamin which prevents the peroxidation of PUFA $^{(60)}$. The mechanism of vitamin $\mathrm{E}$ involves endogenous arachidonic acid release and its conversion to prostaglandins by affecting phospholipase $\mathrm{A}_{2}$ and COX. Activation of phospholipase $\mathrm{A}_{2}$ is considered to be regulated by protein kinase $\mathrm{C}$ and the increase in the concentration of intracellular Ca. Vitamin $\mathrm{E}$ was found to inhibit protein kinase $C$ in the bovine brain ${ }^{(60)}$. It should be used cautiously in women who are on anticoagulants because vitamin $\mathrm{E}$ can have antiplatelet properties and daily intake should be limited to 400 IU or less ${ }^{(60,61)}$. Vitamin $\mathrm{E}$ is available as a synthetic (d-I- $\alpha$-tocopherol, also referred to as $\alpha$-tocopherol or SRR-tocopherol) formulation. The natural formulation is more bioavailable and, thus, greater in potency by a ratio of $1 \cdot 36$ to $2: 1^{(62)}$. Moreover, $400 \mathrm{IU}$ of natural vitamin $\mathrm{E}$ is not equivalent to $400 \mathrm{IU}$ of synthetic vitamin $\mathrm{E}$.

At present, only one randomised controlled trial has been designed to evaluate the effectiveness for the outcomes of chronic painful symptoms and quality of life of dietary therapy compared with hormonal suppression treatment or placebo after conservative surgery for symptomatic endometriosis stage III-IV (Revised American Fertility Society (r-AFS) classification) ${ }^{(63)}$. A total of 222 consecutive women who underwent conservative pelvic surgery were initially randomised to receive a placebo, treatment only as a control, or a post-operative adjunctive hormonal therapy with gonadotrophin-releasing hormone analogue (GnRH-a) or with continuous low-dose monophasic oral contraceptive or dietary therapy for 6 months. Dietary therapy was a protocol consisting of nutritional intake in addition to vitamins (vitamins $\mathrm{B}_{6}, \mathrm{~A}, \mathrm{C}, \mathrm{E}$ ), mineral salts (Ca, Mg, Se, Zn, Fe), VSL3 lactic ferments (a compound containing Bifidobacterium breve, B. longum, B. infantis, Lactobacillus acidophilus, L. casei, L. bulgaricus and Steptococcus thermophilus), and fish oil, giving an energy intake between 1600 and $2000 \mathrm{kcal}$ (6690 and $8370 \mathrm{~kJ}$ ). The post-operative hormonal suppression therapy and nutritional supplementation groups had less CPP than the control group at 12 months' follow-up $(P<0.001)$. As to the outcome of control pain in the patients treated with dietary supplementation, compared with post-operative hormonal therapy, both therapies were similarly effective in reducing painful symptoms.

So, agents with antioxidant activity are able to improve endometriosis-related symptoms, without undesired prolonged hypo-oestrogenism state effects and any important metabolic change of hormonal suppression therapy.

Moreover, a recent study suggests that specific types of dietary fat such as trans-unsaturated fat are associated with the incidence of laparoscopically confirmed endometriosis, and that these relationships may indicate modifiable risk $^{(64)}$. This evidence additionally provides another disease association that supports efforts to remove trans-fat from hydrogenated oils from the food supply.

Sesti et al. provide evidence that post-operative hormonal and dietary therapies were the most effective treatment, not only in terms of painful symptoms control, but also in terms of general health perception and vitality, when compared with post-operative placebo administration ${ }^{(63)}$. Conversely, the same authors showed that a 6-month course of dietary therapy or hormonal suppression treatment after laparoscopic cystectomy had no significant effect on the recurrence rate of ovarian endometriosis when compared with surgery plus placebo ${ }^{(65)}$. Further randomised trials with larger series and long-term followup to confirm these observations are needed.

\section{Conclusions}

CPP is a prevalent problem in women with healthcare costs associated. Women who suffer from CPP are a heterogeneous group, and the possible diagnosis, treatment and contributing factors are varied and complex.

After looking for the most common causes and ruling out serious disease, the approach of treating either the underlying condition or the pain itself or both is effective. It allows the use of pain-directed therapies that, albeit not curative, permit the patient to progress toward a more satisfactory quality of life that is not dominated by pain. In every case, a multidisciplinary and integrative approach may offer expanded therapeutic solutions that are beneficial for some outcome measures.

At present, the experience of medical or surgical interventions for treating CPP is expansive; however, randomised controlled trials performed to evaluate other medical interventions are few and unsatisfactory. In this context, dietary supplements with antioxidants could be considered as a new effective strategy in the long term for CPP; these, not being limited in their use by time, can be used in longterm therapy and may be better accepted by patients.

So, it is to be hoped that urgently required research in the future will identify complementary therapies and evaluate their efficacy in treating CPP.

\section{Acknowledgements}

This research received no specific grant from any funding agency in the public, commercial or not-for-profit sectors.

The contribution of each author to the paper was as follows: F. S. was involved in study design and in drafting of the manuscript; T. C. was involved in the review of the 
literature and writing of the manuscript; A. P. was involved in English language and style revision; M. C. was involved in the review of the literature; M. R. B. was involved in data collection; E. P. was involved with the final corrections.

There are no conflicts of interest.

\section{References}

1. Harris RD, Holtzman SR \& Poppe AM (2000) Clinical outcome in female patients with pelvic pain and normal pelvic US findings. Radiology 216, 440-443.

2. Romão AP, Gorayeb R, Romão GS, et al. (2009) High levels of anxiety and depression have a negative effect on quality of life of women with chronic pelvic pain. Int J Clin Pract 63, 707-711.

3. Vercellini P, Somigliana E, Viganò P, et al. (2009) Chronic pelvic pain in women: etiology, pathogenesis and diagnostic approach. Gynecol Endocrinol 25, 149-158.

4. Poleshuck EL, Bair MJ, Kroenke K, et al. (2009) Pain and depression in gynecology patients. Psychosomatics 50, $270-276$.

5. Sepulcri R, de P \& do Amaral VF (2009) Depressive symptoms, anxiety, and quality of life in women with pelvic endometriosis. Eur J Obstet Gynecol Reprod Biol 142, 53-56.

6. American College of Obstetricians and Gynecologists (2004) Practice Bulletin no. 51. Chronic pelvic pain. Obstet Gynecol 130, 589-605.

7. Sharma D, Dahiya K, Duhan N, et al. (2010) Diagnostic laparoscopy in chronic pelvic pain Arch Gynecol Obstet, (epublication ahead of print version 14 January 2010).

8. Randolph ME \& Reddy DM (2006) Sexual abuse and sexual functioning in a chronic pelvic pain sample. J Child Sex Abus 15, 61-78.

9. Zondervan KT, Yudkin PL, Vessey MP, et al. (2001) Chronic pelvic pain in the community: symptoms, investigations, and diagnoses. Am J Obstet Gynecol 184, 1149-1155.

10. Aslam N, Harrison G, Khan K, et al. (2009) Visceral hyperalgesia in chronic pelvic pain. Br J Obstet Gynecol 116, 1551-1555.

11. Malykhina AP (2007) Neural mechanisms of pelvic organ cross-sensitization. Neuroscience 149, 660-672.

12. Neis KJ \& Neis F (2009) Chronic pelvic pain: cause, diagnosis and therapy from a gynaecologist's and an endoscopist's point of view. Gynecol Endocrinol 25, 757-761.

13. Howard FM (1996) The role of laparoscopy in the evaluation of chronic pelvic pain: pitfalls with a negative laparoscopy. J Am Assoc Gynecol Laparosc 4, 85-94.

14. Cervero F \& Laird JM (1999) Visceral pain. Lancet 353, $2145-2148$.

15. Gebhart GF (2000) J.J. Bonica Lecture - 2000: Physiology, pathophysiology, and pharmacology of visceral pain. Reg Anesth Pain Med 25, 632-638.

16. Buéno L, Fioramonti J \& Garcia-Villar R (2000) Pathobiology of visceral pain: molecular mechanisms and therapeutic implications. III. Visceral afferent pathways: a source of new therapeutic targets for abdominal pain. Am J Physiol Gastrointest Liver Physiol 278, G670-G676.

17. Abbott J, Hawe J, Hunter D, et al. (2004) Laparoscopic excision of endometriosis: a randomized, placebo-controlled trial. Fertil Steril 82, 878-884.

18. Whiteside JL \& Falcone $\mathrm{T}$ (2003) Endometriosis-related pelvic pain: what is the evidence? Clin Obstet Gynecol $\mathbf{4 6}$, $824-830$.
19. Sharpe-Timms KL (2002) Using rats as a research model for the study of endometriosis. Ann N Y Acad Sci 955 318-327, 340-342, 396-406.

20. Berkley KJ, Rapkin AJ \& Papka RE (2005) The pains of endometriosis. Science 308, 1587-1589.

21. Kyama CM, Mihalyi A, Simsa P, et al. (2008) Non-steroidal targets in the diagnosis and treatment of endometriosis. Curr Med Chem 15, 1006-1017.

22. Thacker MA, Clark AK, Marchand F, et al. (2007) Pathophysiology of peripheral neuropathic pain: immune cells and molecules. Anesth Analg 105, 838-847.

23. Lai M, Lü B, Xing X, et al. (2006) Secretagogin, a novel neuroendocrine marker, has a distinct expression pattern from chromogranin A. Virchows Arch 449, 402-409.

24. Arici A, Oral E, Attar E, et al. (1997) Monocyte chemotactic protein-1 concentration in peritoneal fluid of women with endometriosis and its modulation of expression in mesothelial cells. Fertil Steril 67, 1065-1072.

25. Sommer C \& Kress M (2004) Recent findings on how proinflammatory cytokines cause pain: peripheral mechanisms in inflammatory and neuropathic hyperalgesia. Neurosci Lett 361, 184-187.

26. Wu MY \& Ho HN (2003) The role of cytokines in endometriosis. Am J Reprod Immunol 49, 285-296.

27. Anaf V, Simon P, El Nakadi I, et al. (2002) Hyperalgesia, nerve infiltration and nerve growth factor expression in deep adenomyotic nodules, peritoneal and ovarian endometriosis. Hum Reprod 17, 1895-1900.

28. Matsuzaki S, Canis M, Pouly JL, et al. (2004) Cyclooxygenase2 expression in deep endometriosis and matched eutopic endometrium. Fertil Steril 82, 1309-1315.

29. Lousse J-C, Defrère S, Colette $S$, et al. (2010) Expression of eicosanoid biosynthetic and catabolic enzymes in peritoneal endometriosis. Hum Reprod 25, 734-741.

30. Howard FM (2009) Endometriosis and mechanisms of pelvic pain. J Minim Invasive Gynecol 16, 540-550.

31. Rudick CN, Chen MC, Mongiu AK, et al. (2007) Organ cross talk modulates pelvic pain. Am J Physiol Regul Integr Comp Physiol 293, R1191-R1198.

32. Atwal G, du Plessis D, Armstrong G, et al. (2005) Uterine innervation after hysterectomy for chronic pelvic pain with, and without, endometriosis. Am J Obstet Gynecol 193, $1650-1655$.

33. Agarwal A, Gupta S \& Sharma RK (2005) Role of oxidative stress in female reproduction. Reprod Biol Endocrinol 3, 28.

34. Szczepanska M, Kozlik J, Skrzypczak J, et al. (2003) Oxidative stress may be a piece in the endometriosis puzzle. Fertil Steril 79, 1288-1293.

35. King AE, Critchley HOD \& Kelly RW (2001) The NF-кB pathway in human endometrium and first trimester decidua. Mol Hum Reprod 7, 175-183.

36. Evans MD, Dizdaroglu M \& Cooke MS (2004) Oxidative DNA damage and disease: induction, repair and significance. Mutat Res 567, 1-61.

37. Giudice LC \& Kao LC (2004) Endometriosis. Lancet 364 1789-1799.

38. Ota H, Igarashi S, Hatazawa J, et al. (1999) Endometriosis and free radicals. Gynecol Obstet Invest 48, 29-35.

39. Osborn BH, Haney AF, Misukonis MA, et al. (2002) Inducible nitric oxide synthase expression by peritoneal macrophages in endometriosis-associated infertility. Fertil Steril 77, 46-51.

40. Dong M, Shi Y, Cheng Q, et al. (2001) Increased nitric oxide in peritoneal fluid from women with idiopathic infertility and endometriosis. J Reprod Med 46, 887-891.

41. Shanti A, Santanam N, Morales AJ, et al. (1999) Autoantibodies to markers of oxidative stress are elevated in women with endometriosis. Fertil Steril 71, 1115-1118. 
42. Foyouzi N, Berkkanoglu M, Arici A, et al. (2004) Effects of oxidants and antioxidants on proliferation of endometrial stromal cells. Fertil Steril 82, 1019-1022.

43. Kobayashi H, Yamada Y, Kanayama S, et al. (2009) The role of iron in the pathogenesis of endometriosis. Gynecol Endocrinol 25, 39-52.

44. Gambone JC, Mittman BS, Munro MG, et al. (2002) Chronic Pelvic Pain/Endometriosis Working Group. Consensus statement for the management of chronic pelvic pain and endometriosis: proceedings of an expert-panel consensus process. Fertil Steril 78, 961-972.

45. Howard FM (2003) Chronic pelvic pain. Obstet Gynecol 101, 594-611.

46. Stones RW \& Mountfield J (2000) Interventions for treating chronic pelvic pain in women. Cochrane Database of Systematic Reviews, issue 2, CD000387. http://www.mrw. interscience.wiley.com/cochrane/clsysrev/articles/CD000387/ frame.html

47. Indraccolo U \& Barbieri F (2010) Effect of palmitoylethanolamide-polydatin combination on chronic pelvic pain associated with endometriosis: preliminary observations. Eur $J$ Obstet Gynecol Reprod Biol 150, 76-79.

48. Facci L, Dal Toso R, Romanello S, et al. (1995) Mast cells express a peripheral cannabinoid receptor with differential sensitivity to anandamide and palmitoylethanolamide. Proc Natl Acad Sci U S A 92, 3376-3380.

49. Zhao KS, Jin C, Huang X, et al. (2003) The mechanism of Polydatin in shock treatment. Clin Hemorbeol Microcirc 29, 211-217.

50. Herbert B (2010) Chronic pelvic pain. Altern Ther Health Med 16, 28-33.

51. Parazzini F, Chiaffarino F, Surace M, et al. (2004) Selected food intake and risk of endometriosis. Hum Reprod 19 , 1755-1759.

52. Institute of Medicine of the National Academics (2005) Complementary and Alternative Medicine in the United States. Washington, DC: National Academics Press.

53. Proctor M \& Murphy PA (2001) Herbal and dietary therapies for primary and secondary dysmenorrhoea. Cochrane Database of Systematic Reviews, issue 2, CD002124. http://www. mrw.interscience.wiley.com/cochrane/clsysrev/articles/CD0 02124/frame.html
54. Barnard ND, Scialli AR, Hurlock D, et al. (2000) Diet and sexhormone binding globulin dysmenorrhoea and premenstrual symptoms. Obstet Gynecol 95, 245-250.

55. Ziaei S, Zakeri M \& Kazemnejad A (2005) A randomised controlled trial of vitamin $\mathrm{E}$ in the treatment of primary dysmenorrhoea. Br J Obstet Gynecol 112, 466-469.

56. Kidd PM (2007) Omega-3 DHA and EPA for cognition, behavior, and mood: clinical findings and structural-functional synergies with cell membrane phospholipids. Altern Med Rev 12, 207-227.

57. Halliwell B \& Gutteridge JM (1990) Role of free radicals and catalytic metal ions in human disease: an overview. Methods Enzymol 186, 1-85.

58. Alberts B, Johnson A, Lewis J, et al. (2002) Molecular Biology of the Cell, 4th ed. New York, NY: Garland Science.

59. Harel Z, Biro FM, Kottenhahn RK, et al. (1996) Supplementation with omega-3 polyunsaturated fatty acids in the management of dysmenorrhea in adolescents. Am J Obstet Gynecol 174, 1335-1338.

60. Dennehy CE (2006) The use of herbs and dietary supplements in gynecology: an evidence-based review. $J$ Midwifery Womens Health 51, 402-409.

61. Ziaei S, Faghihzadeh S, Sohrabvand F, et al. (2001) A randomised placebo-controlled trial to determine the effect of vitamin $\mathrm{E}$ in treatment of primary dysmenorrhoea. $\mathrm{Br} J$ Obstet Gynecol 108, 1181-1183.

62. Ford ES, Ajani UA \& Mokdad AH (2005) Brief communication: the prevalence of high intake of vitamin $\mathrm{E}$ from the use of supplements among US adults. Ann Inter Med 29, 31-35.

63. Sesti F, Pietropolli A, Capozzolo T, et al. (2007) Hormonal suppression treatment or dietary therapy versus placebo in the control of painful symptoms after conservative surgery for endometriosis stage III-IV. A randomized comparative trial. Fertil Steril 88, 1541-1547.

64. Missmer SA, Chavarro JE, Malspeis S, et al. (2010) A prospective study of dietary fat consumption and endometriosis risk. Hum Reprod 25, 1528-1535.

65. Sesti F, Capozzolo T, Pietropolli A, et al. (2009) Recurrence rate of endometrioma after laparoscopic cystectomy: a comparative randomized trial between post-operative hormonal suppression treatment or dietary therapy vs. placebo. Eur J Obstet Gynecol Reprod Biol 147, 72-77. 